\title{
On Maximal Regularity and Semivariation of $\alpha$-Times Resolvent Families*
}

\author{
Fubo Li, Miao Li \\ Department of Mathematics, Sichuan University, Chengdu, China \\ Email: lifubo@scu.edu.cn,mli@scu.edu.cn
}

Received October 15, 2013; revised November 15, 2013; accepted November 21, 2013

Copyright (C) 2013 Fubo Li, Miao Li. This is an open access article distributed under the Creative Commons Attribution License, which permits unrestricted use, distribution, and reproduction in any medium, provided the original work is properly cited.

\section{ABSTRACT}

Let $1<\alpha<2$ and $A$ be the generator of an $\alpha$-times resolvent family $\left\{S_{\alpha}(t)\right\}_{t>0}$ on a Banach space $X$. It is shown that the fractional Cauchy problem $\mathbf{D}_{t}^{\alpha} u(t)=A u(t)+f(t), t \in(0, r] ; u(0), u^{\prime}(0) \in D(A)$ has maximal regularity on $C([0, r] ; X)$ if and only if $S_{\alpha}(\cdot)$ is of bounded semivariation on $[0, r]$.

Keywords: $\alpha$-Times Resolvent Family; Maximal Regularity; Semivariation

\section{Introduction}

Many initial and boundary value problems can be reduced to an abstract Cauchy problem of the form

$$
\begin{aligned}
& u^{\prime}(t)=A u(t)+f(t), t \in[0, r] \\
& u(0)=x \in D(A)
\end{aligned}
$$

where $A$ is the generator of a $C_{0}$-semigroup. One says that (1.1) has maximal regularity on $C([0, r] ; X)$ if for every $f \in C([0, r] ; X)$ there exists a unique $u \in C^{1}([0, r] ; X)$ satisfying (1.1). From the closed graph theorem it follows easily that if there is maximal regularity on $C([0, r] ; X)$, then there exists a constant $C>0$ such that

$$
\left\|u^{\prime}\right\|_{C([0, r] ; X)}+\|A u\|_{C([0, r] ; X)} \leq\|f\|_{C([0, r] ; X)} .
$$

Travis [1] proved that the maximal regularity is equivalent to the $C_{0}$-semigroup generated by $A$ being of bounded semivariation on $[0, r]$.

Chyan, Shaw and Piskarev [2] gave similar results for second order Cauchy problems. More precisely, they showed that the second order Cauchy problem

$$
\begin{aligned}
& u^{\prime \prime}(t)=A u(t)+f(t), t \in(0, r] \\
& u(0)=x, u^{\prime}(0)=y, \quad x, y \in D(A)
\end{aligned}
$$

has maximal regularity on $[0, r]$ if and only if the cosine

${ }^{*}$ The authors are partially supported by the NSFC of China (Grant No. 11371263), and the second author is also supported by Program for New Century Excellent Talents in University of China. opeator function generated by $A$ is of bounded semivariation on $[0, r]$.

In this paper, we will consider the maximal regularity for fractional Cauchy problem

$$
\begin{aligned}
& \mathbf{D}_{t}^{\alpha} u(t)=A u(t)+f(t), t \in(0, r] \\
& u(0)=x, u^{\prime}(0)=y, \quad x, y \in D(A)
\end{aligned}
$$

where $\alpha \in(1,2), A$ is the generator of an $\alpha$-times resolvent family (see Definition 2.2) and $\mathbf{D}_{t}^{\alpha} u$ is understood in the Caputo sense. We show that (1.3) has maximal regularity on $C([0, r] ; X)$ if and only if the corresponding $\alpha$-times resolvent family is of bounded semivariation on $[0, r]$.

\section{Preliminaries}

Let $1<\alpha<2, \quad g_{0}(t):=\delta(t)$ and

$$
g_{\beta}(t):=\frac{t^{\beta-1}}{\Gamma(\beta)}(\beta>0)
$$

for $t>0$. Recall the Caputo fractional derivative of order $\alpha>0$

$$
\mathbf{D}_{t}^{\alpha} f(t):=\int_{0}^{t} g_{2-\alpha}(t-s) \frac{\mathrm{d}^{2}}{\mathrm{~d} s^{2}} f(s) \mathrm{d} s, \quad t \in[0, r]
$$

for $f \in C^{2}([0, r] ; X)$. The condition that

$$
f \in C^{2}([0, r] ; X)
$$


can be relaxed to $f \in C^{1}([0, r] ; X)$ and

$$
g_{2-\alpha} *\left(f-f(0)-f^{\prime}(0) g_{2}\right) \in C^{2}([0, r] ; X),
$$

for details and further properties see [3] and references therein. And in the above we denote by

$$
\left(g_{\beta} * f\right)(t)=\int_{0}^{t} g_{\beta}(t-s) f(s) \mathrm{d} s
$$

the convolution of $g_{\beta}$ with $f$. Note that $g_{\alpha} * g_{\beta}=g_{\alpha+\beta}$.

Consider a closed linear operator $A$ densely defined in a Banach space $X$ and the fractional evolution Equation (1.3).

Definition 2.1 A function $u \in C([0, r] ; X)$ is called a strong solution of (1.3) if

$$
\begin{aligned}
& u \in C([0, r] ; D(A)) \cap C^{1}([0, r] ; X), \\
& g_{2-\alpha} *(u(t)-x-t y) \in C^{2}([0, r] ; X)
\end{aligned}
$$

and (1.3) holds on $[0, r] . u \in C([0, r] ; X)$ is called a mild solution of (1.3) if $g_{\alpha} * u \in D(A)$ and

$$
u(t)-x-t y=A\left(g_{\alpha} * u\right)(t)+\left(g_{\alpha} * f\right)(t)
$$

for $t \in[0, r]$.

Definition 2.2 Assume that $A$ is a closed, densely defined linear operator on $X$. A family $\left\{S_{\alpha}(t)\right\}_{t \geq 0} \subset B(X)$ is called an $\alpha$-times resolvent family generated by $A$ if the following conditions are satisfied:

(a) $S_{\alpha}(\cdot)$ is strongly continuous on $\mathbb{R}_{+}$and $S_{\alpha}(0)=I$;

(b) $S_{\alpha}(t) D(A) \subset D(A)$ and $A S_{\alpha}(t) x=S_{\alpha}(t) A x$ for all $x \in D(A), t \geq 0$;

(c) For all $x \in D(A)$ and $t \geq 0$, $S_{\alpha}(t) x=x+\left(g_{\alpha} * S_{\alpha}\right)(t) A x$.

Remark 2.3 Since $A$ is closed and densely defined, it is easy to show that for all $x \in X,\left(g_{\alpha} * S_{\alpha}\right)(t) x \in D(A)$ and $A\left(g_{\alpha} * S_{\alpha}\right)(t) x=S_{\alpha} x-x$.

The $\alpha$-times resolvent families are closely related to the solutions of (1.3). It was shown in [3] that if $A$ generates an $\alpha$-times resolvent family $S_{\alpha}(\cdot)$, then (1.3) has a unique strong solution given by $S_{\alpha}(t) x+\int_{0}^{t} S_{\alpha}(s) y \mathrm{~d} s$.

Next, we recall the definition of functions of bounded semivariation (see e.g. [4]). Given a closed interval $[a, b]$ of the real line, a subdivision of $[a, b]$ is a finite sequence $d: a=d_{0}<d_{1}<\cdots<d_{n}=b$. Let $D[a, b]$ denote the set of all subdivisions of $[a, b]$.

Definition 2.4 For

$$
G:[a, b] \rightarrow B(X) \text { and } d \in D[a, b],
$$

define

$$
\begin{aligned}
& S V_{d}[G] \\
& =\sup \left\{\left\|\sum_{n=1}^{n}\left[G\left(d_{i}\right)-G\left(d_{i-1}\right)\right] x_{i}\right\|: x_{i} \in X,\left\|x_{i}\right\| \leq 1\right\}
\end{aligned}
$$

and

$$
S V[G]=\sup \left\{S V_{d}[G]: d \in D[a, b]\right\} .
$$

We say $G$ is of bounded semivariation if $S V[G]<\infty$.

\section{Main Results}

We begin with some properties on $\alpha$-times resolvent families which will be needed in the sequel.

Proposition 3.1 Let $1<\alpha<2$ and $\left\{S_{\alpha}(t)\right\}_{t \geq 0}$ be the $\alpha$-times resolvent family with generator $A$. Define

$$
\begin{aligned}
P_{\alpha}(t) x & =\left(g_{\alpha-1} * S_{\alpha}\right)(t) x \\
& =\int_{0}^{t} g_{\alpha-1}(t-s) S_{\alpha}(s) x \mathrm{~d} s, \quad x \in X,
\end{aligned}
$$

then the following statements are true.

(a) For every $x \in X, \int_{0}^{t} P_{\alpha}(s) x \mathrm{~d} s \in D(A)$ and

$$
A \int_{0}^{t} P_{\alpha}(s) x \mathrm{~d} s=S_{\alpha}(t) x-x
$$

(b) For every $x \in X, 0 \leq a, b \leq t$,

$$
\int_{a}^{b} s P_{\alpha}(t-s) x \mathrm{~d} x \in D(A)
$$

and

$$
\begin{aligned}
A \int_{a}^{b} s P_{\alpha}(t-s) x \mathrm{~d} s & =a S_{\alpha}(t-a) x-b S_{\alpha}(t-b) x \\
& +\int_{a}^{b} S_{\alpha}(t-s) x \mathrm{~d} s
\end{aligned}
$$

(c) For every $x \in X$,

$$
\int_{0}^{t} g_{\alpha}(t-s) s P_{\alpha}(s) x \mathrm{~d} s \in D(A)
$$

and

$$
\begin{aligned}
& A\left(\int_{0}^{t} g_{\alpha}(t-s) s P_{\alpha}(s) x \mathrm{~d} s\right) \\
= & -\alpha\left(g_{\alpha} * S_{\alpha}\right)(t) x+t P_{\alpha}(t) x ;
\end{aligned}
$$

(d) If $f \in C([0, r] ; X)$, then $g_{\alpha} * S_{\alpha} * f \in D(A)$ and

$$
A\left(g_{\alpha} * S_{\alpha} * f\right)=\left(S_{\alpha}-1\right) * f .
$$

Proof. (a) follows from the fact that

$$
\begin{aligned}
\int_{0}^{t} P_{\alpha}(s) x \mathrm{~d} s & =\left(g_{1} * g_{\alpha-1} * S_{\alpha}\right)(t) x \\
& =\left(g_{\alpha} * S_{\alpha}\right)(t) x \in D(A)
\end{aligned}
$$

and $A\left(g_{\alpha} * S_{\alpha}\right)(t) x=S_{\alpha}(t) x-x$ by Remark 2.3.

(b) By integration by parts we have

$$
\begin{aligned}
& \int_{a}^{b} s P_{\alpha}(t-s) x \mathrm{~d} s=\int_{a}^{b} s \mathrm{~d}_{s}\left[\int_{0}^{s} P_{\alpha}(t-\tau) x \mathrm{~d} \tau\right] \\
& =\int_{a}^{b} s \mathrm{~d}_{s}\left[\left(g_{\alpha} * S_{\alpha}\right)(t-s) x\right] \\
& =-\left.s\left(g_{\alpha} * S_{\alpha}\right)(t-s) x\right|_{a} ^{b}+\int_{a}^{b}\left(g_{\alpha} * S_{\alpha}\right)(t-s) x \mathrm{~d} s \\
& =a\left(g_{\alpha} * S_{\alpha}\right)(t-a) x-b\left(g_{\alpha} * S_{\alpha}\right)(t-b) x \\
& +\int_{a}^{b}\left(g_{\alpha} * S_{\alpha}\right)(t-s) x \mathrm{~d} s,
\end{aligned}
$$


since $\left(g_{\alpha} * S_{\alpha}\right)(t) x \mathrm{~d} s \in D(A)$ by Remark 2.3, operating $A$ on both sides of the above identity gives (b).

(c) follows from the fact that

$$
\begin{aligned}
& \int_{0}^{t} g_{\alpha}(t-s) s P_{\alpha}(s) x \mathrm{~d} s \\
& =\int_{0}^{t} g_{\alpha}(t-s)(s-t) P_{\alpha}(s) x \mathrm{~d} s+t \int_{0}^{t} g_{\alpha}(t-s) P_{\alpha}(s) x \mathrm{~d} s \\
& =-\alpha \int_{0}^{t} g_{\alpha+1}(t-s) P_{\alpha}(s) x \mathrm{~d} s+t\left(g_{\alpha} * P_{\alpha}\right)(t) x \\
& =-\alpha\left(g_{\alpha+1} * P_{\alpha}\right)(t) x+t\left(g_{\alpha} * P_{\alpha}\right)(t) x \\
& =-\alpha\left(g_{\alpha+1} * g_{\alpha-1} * S_{\alpha}\right)(t) x+t\left(g_{\alpha} * g_{\alpha-1} * S_{\alpha}\right)(t) x \\
& =-\alpha\left(g_{\alpha} * g_{\alpha} * S_{\alpha}\right)(t) x+t\left(g_{\alpha-1} * g_{\alpha} * S_{\alpha}\right)(t) x
\end{aligned}
$$

belongs to $D(A)$ and

$$
\begin{aligned}
& A\left(\int_{0}^{t} g_{\alpha}(t-s) s P_{\alpha}(s) x \mathrm{~d} s\right) \\
= & -\alpha\left(g_{\alpha} * A\left(g_{\alpha} * S_{\alpha}\right)\right)(t) x+t\left(g_{\alpha-1} * A\left(g_{\alpha} * S_{\alpha}\right)\right)(t) x \\
= & -\alpha\left(g_{\alpha} *\left(S_{\alpha}-1\right)\right)(t) x+t\left(g_{\alpha-1} *\left(S_{\alpha}-1\right)\right)(t) x \\
= & -\alpha\left(g_{\alpha} * S_{\alpha}\right)(t) x+\alpha g_{\alpha+1}(t) x \\
& +t\left(g_{\alpha-1} * S_{\alpha}\right)(t)-t g_{\alpha}(t) x \\
= & -\alpha\left(g_{\alpha} * S_{\alpha}\right)(t) x+t P_{\alpha}(t) x .
\end{aligned}
$$

(d) (3.1) is true for step functions, and then for continuous functions by the closedness of $A$.

The following two lemmas can be proved similarly as that in $[1,2]$.

Lemma 3.2 If $f \in C([0, r] ; X)$ and the $\alpha$-times resolvent family $S_{\alpha}(t)$ is of bounded semivariation on $[0, r]$, then $\left(P_{\alpha} * f\right)(t) \in D(A)$ and

$$
A\left(P_{\alpha} * f\right)(t)=-\int_{0}^{t} \mathrm{~d}_{s}\left[S_{\alpha}(t-s)\right] f(s) .
$$

Lemma 3.3 If $f \in C([0, r] ; X)$ and the $\alpha$-times resolvent family $S_{\alpha}(t)$ is of bounded semivariation on $[0, r]$, then $\int_{0}^{t} \mathrm{~d}_{s}\left[S_{\alpha}(t-s)\right] f(s)$ is continuous in $t$ on $[0, r]$.

We next turn to the solution of

$$
\begin{aligned}
& \mathbf{D}_{t}^{\alpha} u(t)=A u(t)+f(t), \quad t \in(0, r], \\
& u(0)=0, u^{\prime}(0)=0,
\end{aligned}
$$

where $A$ is the generator of an $\alpha$-times resolvent family. If $v(t)$ is a mild solution of (3.2), then by Definition $2.1\left(g_{\alpha} * v\right)(t) \in D(A)$ and

$$
v(t)=A\left(g_{\alpha} * v\right)(t)+\left(g_{\alpha} * f\right)(t) .
$$

It then follows from the properties of $\alpha$-times resolvent family that

$$
\begin{aligned}
1 * v & =\left(S_{\alpha}-A\left(g_{\alpha} * S_{\alpha}\right)\right) * v \\
& =S_{\alpha} * v-S_{\alpha} * A\left(g_{\alpha} * v\right) \\
& =S_{\alpha} *\left(v-A\left(g_{\alpha} * v\right)\right) \\
& =S_{\alpha} * g_{\alpha} * f,
\end{aligned}
$$

which implies that $g_{\alpha} * S_{\alpha} * f$ is differentiable and

$$
\begin{aligned}
v(t) & =\frac{\mathrm{d}}{\mathrm{d} t}\left(g_{\alpha} * S_{\alpha} * f\right)(t) \\
& =\left(g_{\alpha-1} * S_{\alpha} * f\right)(t) \\
& =\left(P_{\alpha} * f\right)(t) .
\end{aligned}
$$

Therefore, the mild solution of (1.3) is given by

$$
u(t)=S_{\alpha}(t) x+\int_{0}^{t} S_{\alpha}(s) y \mathrm{~d} s+\left(P_{\alpha} * f\right)(t) .
$$

Proposition 3.4 Let A be the generator of an $\alpha$-times resolvent family $S_{\alpha}(\cdot)$, and let $f \in C([0, r] ; X)$ and $x, y \in D(A)$. Then the following statements are equivalent:

(a) (1.3) has a strong solution;

(b) $\left(S_{\alpha} * f\right)(\cdot) \in C^{1}([0, r] ; X)$;

(c) $\left(P_{\alpha} * f\right)(t) \in D(A)$ for $0 \leq t \leq r$ and $A\left(P_{\alpha} * f\right)(t)$ is continuous in $t$ on $[0, r]$.

Proof. (a) If $u(t)$ is a strong solution of (1.3), then $u$ is given by (3.3) since every strong solution is a mild solution. Therefore, by the definition of strong solutions,

$$
g_{2-\alpha} * P_{\alpha} * f=g_{1} * S_{\alpha} * f \in C^{2}([0, r] ; X) ;
$$

it then follows that $S_{\alpha} * f \in C^{1}([0, r] ; X)$, this is (b).

(b) $\Rightarrow$ (c). Suppose that $S_{\alpha} * f \in C^{1}([0, r] ; X)$. Since $g_{1} * P_{\alpha} * f=g_{\alpha} * S_{\alpha} * f$, by Proposition 3.1(d),

$$
g_{1} * P_{\alpha} * f \in D(A)
$$

and

$$
A\left(g_{1} * P_{\alpha} * f\right)=A\left(g_{\alpha} * S_{\alpha} * f\right)=\left(S_{\alpha}-1\right) * f .
$$

Since $A$ is closed and $S_{\alpha} * f \in C^{1}([0, r] ; X)$, we have $P_{\alpha} * f \in D(A)$ and $A\left(P_{\alpha} * f\right)=\left(S_{\alpha} * f\right)^{\prime}-f$ is continuous.

(c) $\Rightarrow$ (a). By (3.4),

$$
g_{1} * A\left(P_{\alpha} * f\right)=A\left(g_{1} * P_{\alpha} * f\right)=\left(S_{\alpha}-1\right) * f,
$$

therefore $S_{\alpha} * f$ is differentiable and thus

$$
g_{2-\alpha} * P_{\alpha} * f=g_{1} * S_{\alpha} * f
$$

is in $C^{2}([0, r] ; X)$. It is easy to check that $u(t)$ defined by (3.3) is a strong solution of (1.3).

Now we are in the position to give the main result of this paper. The proof is similar to that of Proposition 3.1 in [1] or Theorem 4.2 in [2], we write it out for completeness. 
Theorem 3.5 Suppose that $A$ generates an $\alpha$-times resolvent family $\left\{S_{\alpha}(t)\right\}_{t \geq 0}$. Then the function (3.3) is a strong solution of the Cauchy problem (1.3) for every pair $x, y \in D(A)$ and continuous function $f$ if and only if $S_{\alpha}(\cdot)$ is of bounded semivariation on $[0, r]$.

Proof. The sufficiency follows from Lemmas 3.2 and 3.3.

Conversely, suppose that for $x, y \in D(A)$ and continuous function $f, u(t)$ given by (3.3) is a strong solution for (1.3). Define the bounded linear operator $L: C([0, r] ; X) \rightarrow X$ by $L(f)=\left(P_{\alpha} * f\right)(r)$. By Pro- position 3.4(c) $L f \in D(A)$, it thus follows from the closedness of $A$ that $A L: C([0, r] ; X) \rightarrow X$ is bounded.

Let $\left\{d_{i}\right\}_{i=0}^{n}$ be a subdivision of $[0, r]$ and $\epsilon>0$ such that $\epsilon<\min _{1 \leq i \leq n}\left\{\left|d_{i}-d_{i-1}\right|\right\}$. For $x_{i} \in X$ with $\left\|x_{i}\right\| \leq 1(i=1,2, \cdots, n+1)$, define $f_{d, \epsilon} \in C([0, r] ; X)$ by

$$
f_{d, \epsilon}(\tau)=\left\{\begin{array}{ll}
x_{i}, & d_{i-1} \leq \tau \leq d_{i}-\epsilon \\
x_{i+1}+\frac{\tau-d_{i}}{\epsilon}\left(x_{i+1}-x_{i}\right), & d_{i}-\epsilon \leq \tau \leq d_{i}
\end{array},\right.
$$

then $\left\|f_{d, \epsilon}\right\|_{C([0, r] ; X)} \leq 1$. By Proposition 3.1,

$$
\begin{aligned}
A L\left(f_{d, \epsilon}\right)= & A \int_{0}^{r} P_{\alpha}(r-s) f_{d, \epsilon}(s) \mathrm{d} s \\
= & \sum_{i=1}^{n}\left[A \int_{d_{i-1}}^{d_{i}-\epsilon} P_{\alpha}(r-s) x_{i} \mathrm{~d} s+A \int_{d_{i}-\epsilon}^{d_{i}} P_{\alpha}(r-s) x_{i+1} \mathrm{~d} s+A \int_{d_{i}-\epsilon}^{d_{i}} \frac{s-d_{i}}{\epsilon} P_{\alpha}(r-s)\left(x_{i+1}-x_{i}\right) \mathrm{d} x\right] \\
= & \sum_{i=1}^{n}\left\{\left[S_{\alpha}\left(r-d_{i-1}\right) x_{i}-S_{\alpha}\left(r-d_{i}+\epsilon\right) x_{i}\right]+\left[S_{\alpha}\left(r-d_{i}+\epsilon\right) x_{i+1}-S_{\alpha}\left(r-d_{i}\right) x_{i+1}\right]\right. \\
& -\frac{d}{\epsilon}\left[S_{\alpha}\left(r-d_{i}+\epsilon\right)\left(x_{i+1}-x_{i}\right)-S_{\alpha}\left(r-d_{i}\right)\left(x_{i+1}-x_{i}\right)\right] \\
& \left.+\frac{1}{\epsilon}\left[\left(d_{i}-\epsilon\right) S_{\alpha}\left(r-d_{i}+\varepsilon\right)\left(x_{i+1}-x_{i}\right)-d_{i} S_{\alpha}\left(r-d_{i}\right)\left(x_{i+1}-x_{i}\right)\right]+\frac{1}{\epsilon} \int_{d_{i}-\epsilon}^{d_{i}} S_{\alpha}(r-s)\left(x_{i+1}-x_{i}\right) \mathrm{d} s\right\} \\
= & \sum_{i=1}^{n}\left\{\left[S_{\alpha}\left(r-d_{i-1}\right) x_{i}-S_{\alpha}\left(r-d_{i}\right) x_{i+1}\right]+\frac{1}{\epsilon} \int_{d_{i}-\epsilon}^{d_{i}} S_{\alpha}(r-s)\left(x_{i+1}-x_{i}\right) \mathrm{d} s\right\} \\
= & \sum_{i=1}^{n}\left\{\left[S_{\alpha}\left(r-d_{i-1}\right)-S_{\alpha}\left(r-d_{i}\right)\right] x_{i}-S_{\alpha}\left(r-d_{i}\right)\left(x_{i+1}-x_{i}\right)+\frac{1}{\epsilon} \int_{d_{i}-\epsilon}^{d_{i}} S_{\alpha}(r-s)\left(x_{i+1}-x_{i}\right) \mathrm{d} s\right\},
\end{aligned}
$$

it then follows that

$$
\left\|\sum_{i=1}^{n}\left[S_{\alpha}\left(r-d_{i-1}\right)-S_{\alpha}\left(r-d_{i}\right)\right] x_{i}\right\| \leq\left\|A L\left(f_{d, \epsilon}\right)\right\|+\sum_{i=1}^{n}\left\|S_{\alpha}\left(r-d_{i}\right)\left(x_{i+1}-x_{i}\right)-\frac{1}{\epsilon} \int_{d_{i}-\epsilon}^{d_{i}} S_{\alpha}(r-s)\left(x_{i+1}-x_{i}\right) \mathrm{d} s\right\| .
$$

By letting $\epsilon \rightarrow 0$, we obtain that $S_{\alpha}$ is of bounded semivariation on $[0, r]$.

Corollary 3.6 Suppose that $\left\{S_{\alpha}(t)\right\}_{t \geq 0}$ is an $\alpha$ times resolvent family with generator $A$ and $S_{\alpha}(\cdot)$ is of bounded semivariation on $[0, r]$ for some $r>0$. Then $R\left(P_{\alpha}(t)\right) \subset D(A)$ for $t \in[0, r]$ and $\left\|t A P_{\alpha}(t)\right\|$ is bounded on $[0, r]$.

Proof. For $x \in X$, consider $f(t)=\alpha S_{\alpha}(t) x$. By Proposition 3.1(c), $t P_{\alpha}(t) x$ is a mild solution of (3.2). Moreover, it follows from Proposition 3.4 that $P_{\alpha} * f$ is a strong solution of (3.2). Since a strong solution must be a mild solution, we have $\left(P_{\alpha} * f\right)(t)=t P_{\alpha}(t) x$. Thus our claim follows from Proposition 3.4.

Remark 3.7 Let $\alpha=1$. If $A$ generates a $C_{0}$-semigroup $T(\cdot)$, then the condition that $\operatorname{tAT}(t)$ is bounded on $[0, r]$ implies that $T(\cdot)$ is analytic (see [5]). When $\alpha=2$ and $A$ generates a cosine function $C(\cdot)$, then the condition that $\operatorname{tAC}(t)$ is bounded on $[0, r]$ implies that
$A$ is bounded ([3]). However, since there is no semigroup properties for $\alpha$-times resolvent family, it is not clear that one can get the analyticity of $S_{\alpha}(\cdot)$ from the local boundedness of $t A P_{\alpha}(t)$.

\section{REFERENCES}

[1] C. C. Travis, "Differentiability of Weak Solutions to an Abstract Inhomogeneous Differential Equation," Proceedings of the American Mathematical Society, Vol. 82, 1981 , pp. 425-430.

http://dx.doi.org/10.1090/S0002-9939-1981-0612734-2

[2] D. K. Chyan, S. Y. Shaw and S. Piskarev, "On Maximal Regularity and Semivariation of Cosine Operator Functions," Journal London Mathematical Society, Vol. 59, No. 3, 1999, pp. 1023-1032. http://dx.doi.org/10.1112/S0024610799007073

[3] E. G. Bajlekova, "Fractional Evolution Equations in Banach Spaces," Dissertation, Eindhoven University of Technology, 2001. 
[4] C. S. Hönig, "Volterra Stieltjes Integral Equations," NorthHolland, Amsterdam, 1975.

[5] A. Pazy, "Semigroups of Linear Operators and Applica- tions to Partial Differential Equations," Springer, New York, 1983. 